\title{
RELATION OF THE GASTRIC CONTENT TO THE SECRETORY AND MOTOR FUNCTIONS OF THE STOMACH *
}

\author{
HOMER WHEELON, M.D.
} ST. LOU]S

From the time of Spallanzani, who was among the first to demonstrate the digestive action of gastric juice, to the present day gastrologist, wide variations of opinion have been held relative to the secretory and motor powers of the stomach. The lack of agreement among students of gastric functions recalls the statement of Hunter: "Some Physiologists will have it that the stomach is a Mill;-others, that it is a fermenting Vat;-others, again, that it is a Stew-pan:-but in my view of the matter, it is neither a Mill, a fermenting Vat, nor a Stew-pan-but a Stomach, Gentlemen, a Stomach."

The "fractional method" of gastric analysis, now so popular among certain clinicians, is based on the assumption that the gastric content is of a homogenous nature. The method also infers that the determination of the acidity of gastric samples removed at stated intervals, more or less, directly show the degree of acid formation by the stomach and that any such sample is indicative of the acid concentration of the entire stomach contents at the time of withdrawal. As stated by Gorham, "If the above hypothysis be a correct one, and based upon a true physiological principle, then we should expect the acid concentration of the different portions of the remaining gastric chyme (content) (Italics are mine) to be similar at these different intervals after a test meal." That such is not the case has been well shown by this observer. To quote: "This hypothysis is not based upon true physiology. The acidity of one portion, as obtained by the fractional method, may differ widely from the acidity of different portions of the remaining contents. In the so-called "fractional," or other methods of gastric analyses when only a small sample is withdrawn, the small portion removed may or may not be representative of the gastric contents remaining in the stomach."

It is my purpose to call attention to certain observations concerning the relation of gastric juice to the ingested food and to present experimental data which corroborates the views of certain of the older

* From the Department of Physiology of the St. Louis University School of Medicine.

1. Gorham, F. D.: Variations of Acid Concentration in Different Portions of the Gastric Chyme, and Its Relation to Clinical Methorls of Gastric Analysis. Arch. Int. Med. 27:434 (April) 1921. 
observers and especially of the recent work of Gorham, namely, that the stomach content is not a homogenous or uniform mixture during the greater portion of the period of gastric digestion.

\section{- METHODS}

The results here reported are based on 296 gastric sample titrations from sixty-four normal medical students. In addition, gastric analyses were made on three men who did not show the presence of free hydrochloric acid. After a thorough examination of the sixty-four men, it was felt that the results obtained could be taken as representative of the average normal for the young male.

In the present work three methods of procedure were employed in determining the acid secretion of the stomach. These methods were: (1) The usual "one hour test" following complete removal of the stomach contents at one time. (2) The "fractional method" or "acid functional test" in which withdrawals of gastric contents were made every fifteen minutes following the ingestion of a test meal. (3) A method recently described by Gorham which consists, in principle, of rapidly removing the entire contents of the stomach in 10 c.c. amounts at a stated time following the taking of a test meal. The third method is here referred to as the "rapid withdrawal method, or test."

In Method 1, the gastric contents was obtained by the use of the large stomach tube at the end of one hour from thirty-one men. In the other two methods a small, soft, stomach tube of the Rehfuss type was passed, the fasting contents removed, and then, with the tube remaining in the stomach, the subjects were given a test meal. In Method 2, "fractional," 10 c.c. quantities of gastric content were withdrawn every fifteen minutes following completion of the meal until the stomach was emptied. This method was used on twenty men; 148 samples were obtained and titrated. In the third method, "rapid withdrawal method," nineteen men were given the tube, and one hour after the test meal the stomach was emptied as rapidly as possible by repeated withdrawals of 10 c.c. amounts. The samples were titrated separately. The nineteen men on whom this method was used returned a total of 117 samples.

Inasmuch as the passage of a stomach tube for the first time is usually associated with more or less retching, salivation, elevation of the blood pressure and nausea, it was deemed advisable to introduce the tube in the second and third methods prior to the ingestion of the meal. Further, it was hoped that such a procedure would permit the tip of the tube to descend into the pyloric portion in which region the concentration of acid is conceded to be highest and the gastric content of a more uniform consistency. That is, an attempt was made to 
obtain results as uniform as possible and to reduce to a minimum the variations in the acid concentration due to withdrawals from various portions of the stomach.

The test meal used throughout this series of experiments consisted of two slices of white bread and 500 c.c. tap water. The meals were given at $S \mathrm{a} . \mathrm{m}$. or following a fast of from ten to twelve hours. The

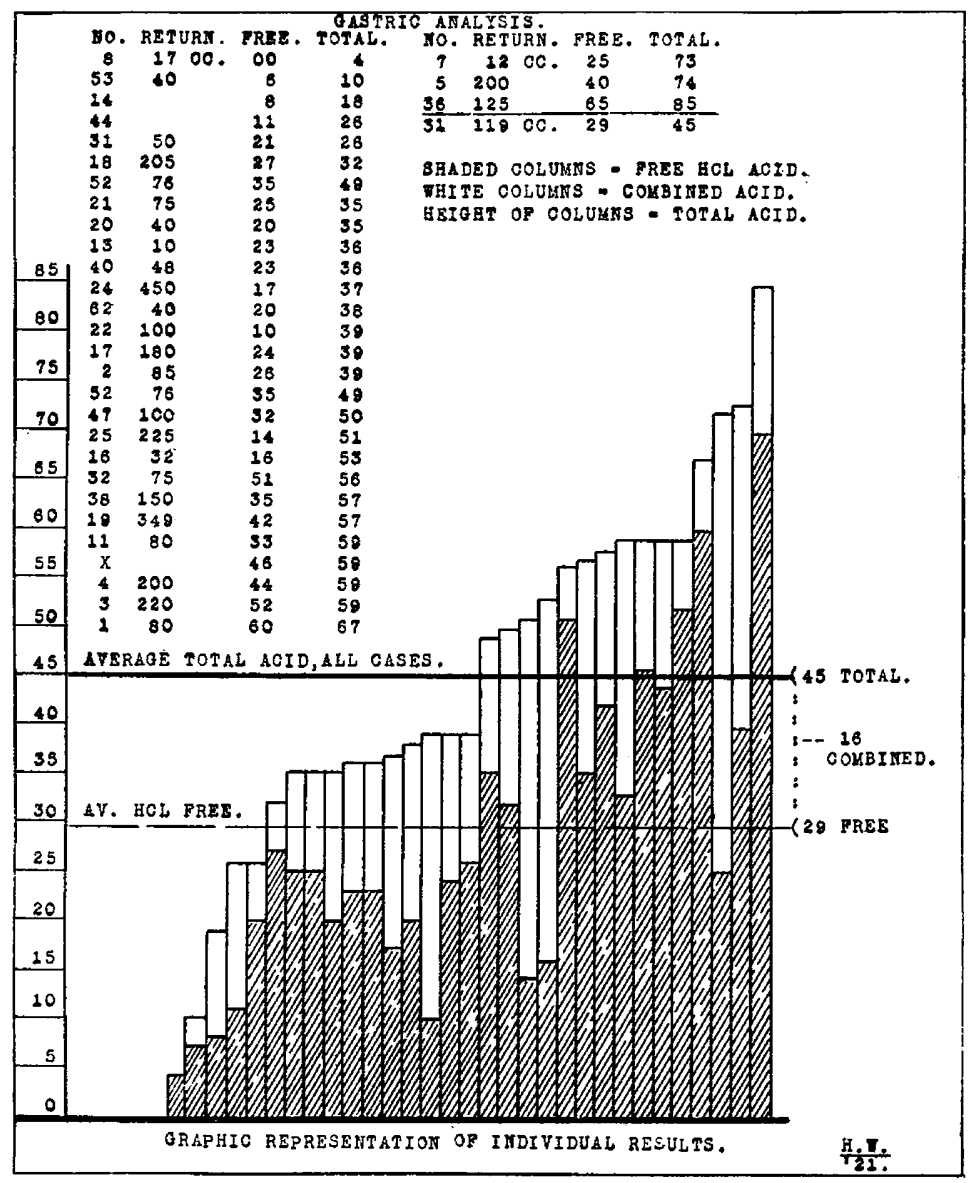

Fig. 1.-Chart showing the individual amounts of gastric return and free and total acids at the end of one hour following the ingestion of a standard test meal. Observations made on 31 young men.

contents of the fasting stomach averaged less than 30 c.c. The acidity of the gastric contents was determined by the Toepfer method of gastric analysis, titrating with tenth-normal sodium hydroxicl solution. Dimethylamidloazobenzol and phenolphthalein were used as indicators. Titrations were made on 10 c.c. samples of gastric content. 


\section{RESULTS}

Results of Experiments by the "One Hour Method."-In these experiments the entire gastric content was received one hour after ingestion of the test meal into a single container. This material was measured then thoroughly mixed and a 10 c.c. sample titrated for free and total acids. The results obtained by such a procedure represent the degree of acidity of the mass as a whole, also the degree of gastric evacuation that had occurred during the hour.

The results of thirty-one experiments of this nature are shown numerically and diagramatically in Figure 1, in which the data are arranged according to the amounts of total acid found. The average total acids for the thirty-one titrations was 45; free acid, 29, combined acid, 16. The range of total acid variation among the thirty-one determinations was from 4 to 85 ; free hydrochloric acid varied from 0 to 65 . The average figures for the entire number of tests lay within the range of "the normal" as generally accepted. It is possible that the first three men of the series show a reduced secretory power of the stomach; however, none of the three had experienced gastric disturbances. Student 8 failed to show the presence of free acid by any one of the three methods employed, also his total acid was continually low.

The average gastric return for twenty-eight men of this group was 119 c.c. The range of variation in returns was great, from 10 to 450 c.c. This point will be considered further in relation to gastric motility.

Results of Experiments by the "Fractional Method."-This method of gastric analysis was studied on twenty-seven men who gave a total return of 183 samples. Of this number of men, twenty were given a test meal and withdrawals were made every fifteen minutes until the stomach was emptied. In seven cases, the men took the tube following a regular breakfast of fruit, cakes, water and coffee. The results on these seven men, while differing but little from those obtained on the group of twenty men, are incomplete for the reason that withdrawals were begun at various time-intervals following the taking of the meal (Table 1).

The numerical results of the observations by the "fractional method" are shown in Table 1, and graphically in Figures 2 and 3. In Table 1 the results are arranged according to the number of samples returned at the various fifteen minute intervals. Of this group of twenty men, one returned fourteen samples-210 minutes; one returned eleven samples; two returned ten samples; two returned nine samples; one returned eight samples; four returned seven samples; four returned six samples; and five returned five samples. The average time of gastric evacuation 


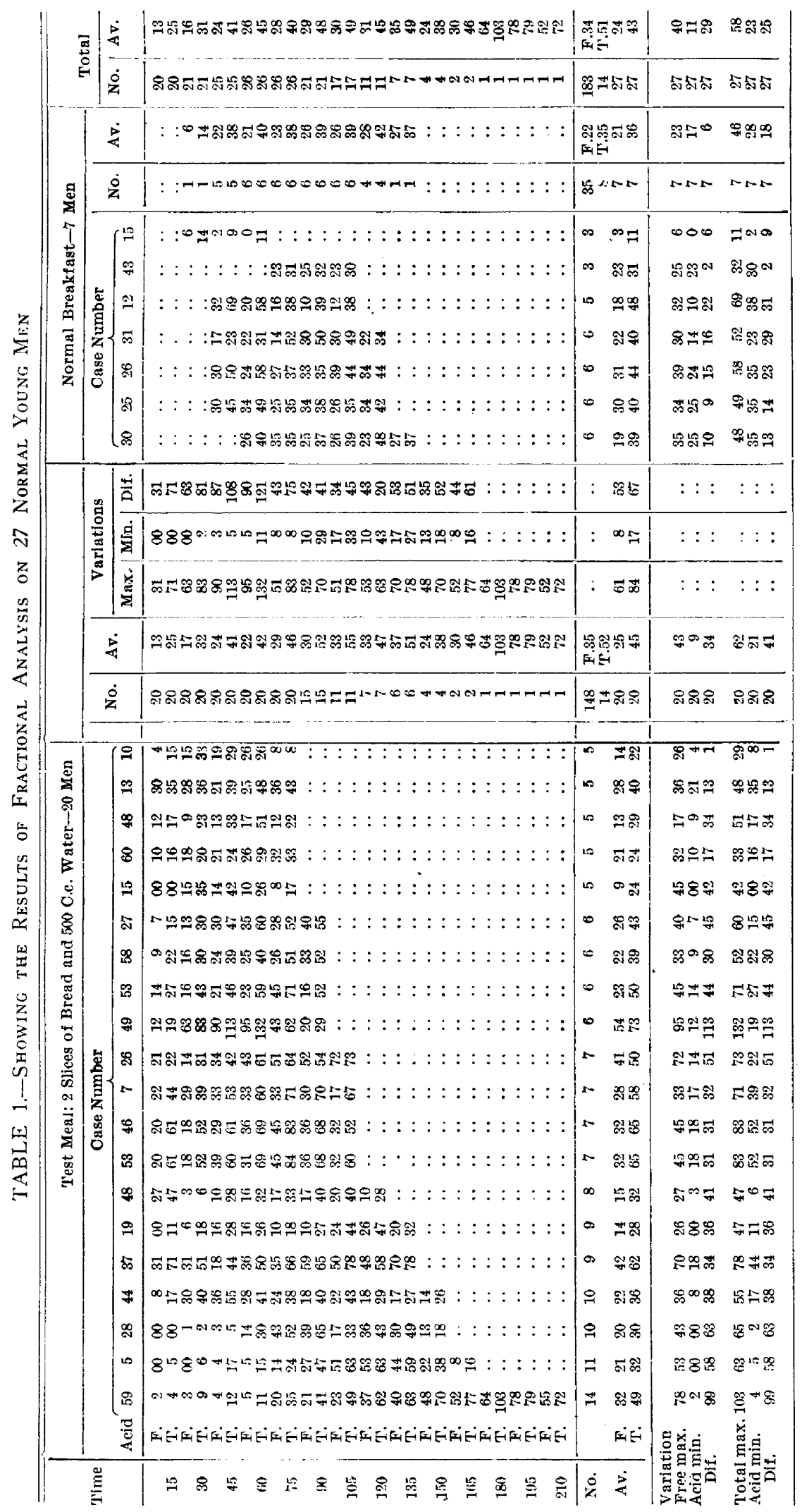

Downloaded From: http://archinte.jamanetwork.com/ by a Oakland University User on 06/03/2015 
for the group was 111 minutes, or an average return of 7.4 samples. The results of titrations on any one man are shown in columns, Table 1 , under the heading Case No. The comparative results for any given time interval are shown by the figures to the right of the time intervals, from 15 to 210 minutes. Averages are shown both for the individual

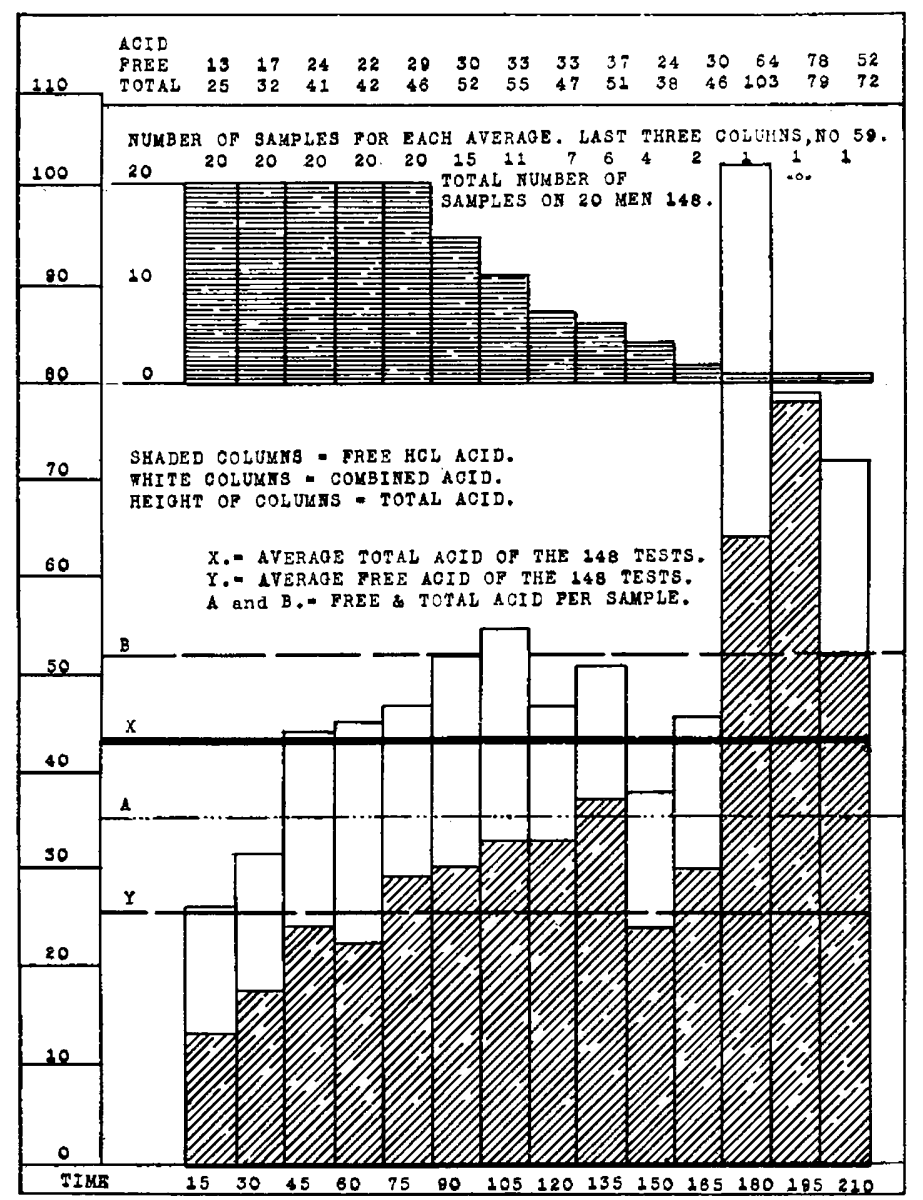

Fig. 2.- Chart showing the collective average of tree and total acid for the entire number of 10 c.c. samples clelivered at 15-minute intervals. "Fractional test meal methorl."

case and for the collective readings at the various time intervals. The range of acid variation is also shown in Table 1.

The relation of the average of all readings for the group of twenty men for any given time period to the number of samples examined is shown in Figure 2. Here, it will be seen that the average free acid for the number of men delivering samples shows a gradual increase up 
to the 135 minute period following the test meal, also that the total acias increase only up to the 105 minute period. The last three columns are the results obtained on one man (No. 59), and, therefore, cannot be taken to indicate a terminal high acid concentration for the group, as a whole. The average free and total acid concentrations for the fourteen time intervals was 35 and 52 , respectively; the average for the entire number of 148 titrations was free acid 25, total 45 . The average variation for acid determinations made on any one individual was free

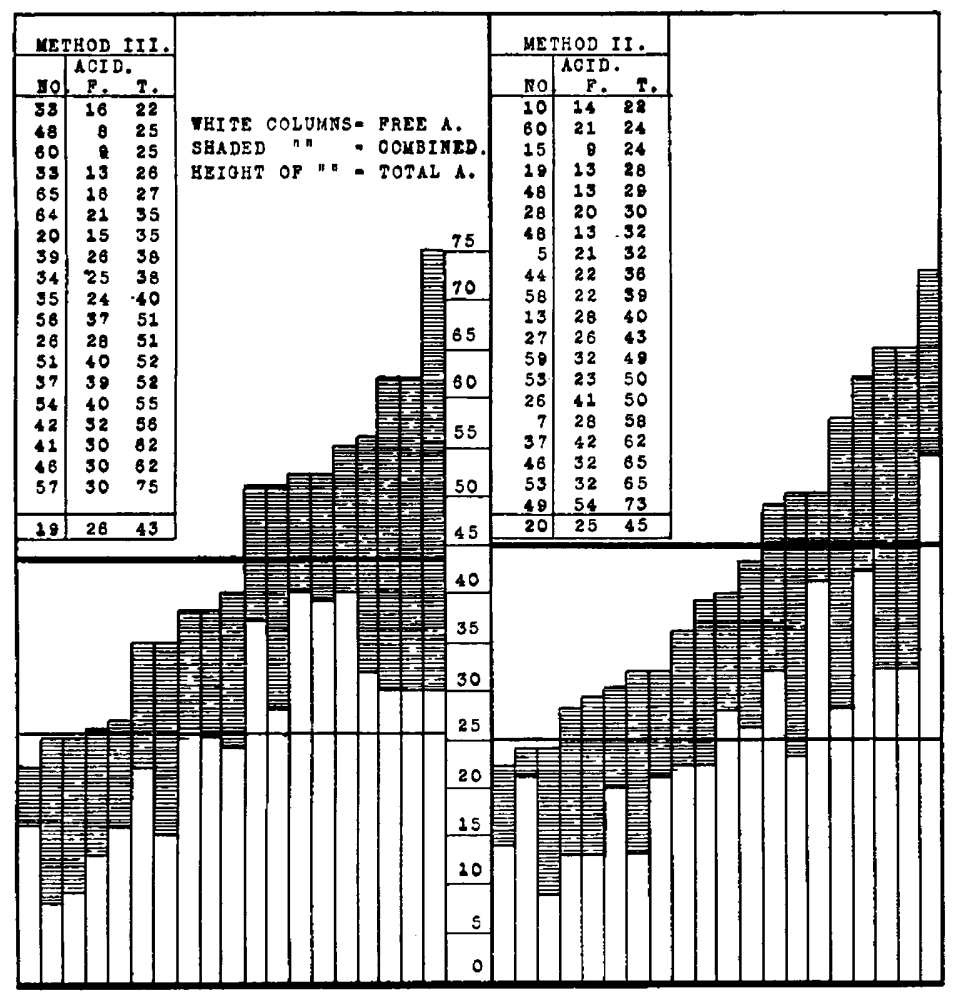

Fig. 3.-Chart showing the individual average free and total acid of the entire number of samples of gastric contents removed in 10 c.c. quantities at the end of one hour "rapid withdrawal method" and at intervals of 15 minutes following a test meal "fractional method."

acid 34, total 41 points. The average variation among titrations for any given time interval was free acid 53 , total 67 points.

In Figure 3 are shown the average free and total acid concentrations for the entire number of samples obtained from any one man. The figures are arranged according to the average total acid concentrations. The difference in the height of the twenty columns indicates the range of variation in the average acid concentrations among the various 
members of the group. It is of interest to note that the average figures for the entire number of titrations for this group of twenty men made over a period of 210 minutes correspond closely with the averages obtained for the first method-total acids 45 against 45 , and free acid 25 against 29 . This appears to be of interest since the height of free acid concentration, as determined by the second method, occurs 135 minutes following the meal, whereas the acid concentration in the first method was determined at the close of one hour. Such results seem to indicate that as much information relative to the secretory power of the stomach is to be gained by the "one hour method" as from the more laborious procedure of the "fractional method."

Results of Experiments by the "Rapid Withdrawal Method."-The primary purpose of this method, as evolved by Gorham, ${ }^{1}$ is the determination of the presence or absence of free acid in any portion of the gastric content at the end of a given period of time. Such a method often reveals the presence of free acid in some one or more of the samples when other methods have failed to show its presence. However, three of the sixty-four students studied, Nos. 63, 29 and 8, did not show the presence of free acid by the first two methods, also failed to show free acid by the rapid withdrawal method. It, therefore, appears that these men had a condition of true achlorhydria.

The reason for the use of this method, as modified for the purpose of this study, was, not to search for free acid in patients, but, simply to determine whether or not the degree of acidity in various portions of the gastric content in normal individuals was uniform, and to compare such results with those obtained by the other two methods. The results of such observations on nineteen normal young men-117 titrationsare shown numerically in Table 2 and graphically in Figure 3 . The data in Table 2 is arranged as in Table 1. In this series of observations two men delivered but two samples $(20$ c. c.) at the end of one hour; three delivered five; nine delivered six; two delivered seven and three men delivered, 9, 10 and 11 samples, respectively. The average free and total acids for the series of eleven samples, as shown in Table 2 , is fairly constant save for the last two columns-samples 10 and 11 . This discrepancy is explainable on the basis of the number of men delivering ten and eleven samples. The average figures for the series of the eleven collective titrations was free acid 26 , total acids 41 . The average figures for the entire number of titrations (117) was free acid 26 , total 43 . The average range of variation among the eleven sets of samples was free acid 34 , total acid 53 points. The average range of variation for the total number of samples delivered by the individual case was free acid 16 points, total 25 . The maximal and minimal variations in the acid concentrations are shown in Table 2. 


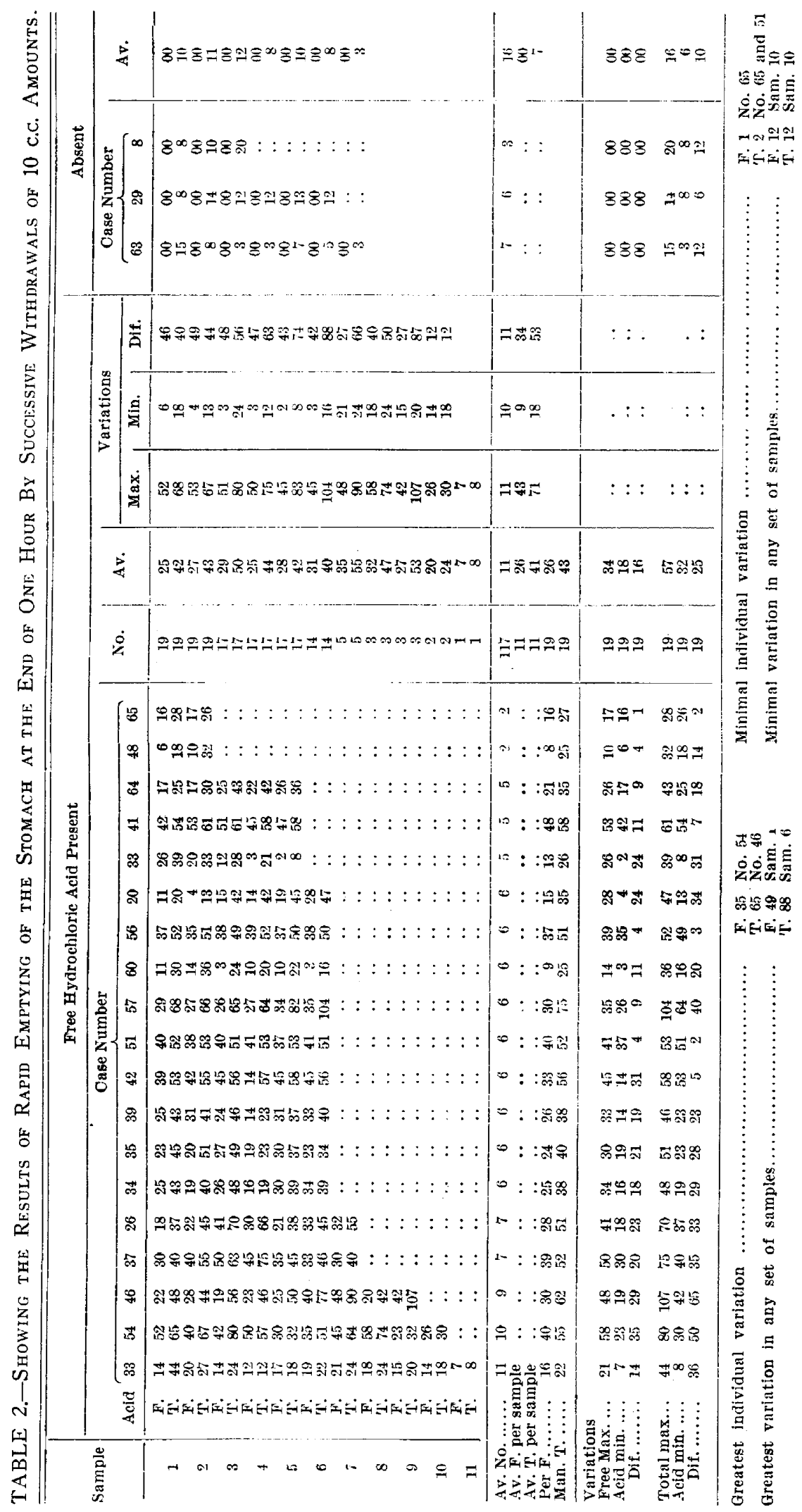


In Figure 3 is shown the average free and total acids for the entire number of samples obtained from any one individual. The average free and total acids for the 117 titrations made on nineteen men, as stated above, was 26 and 43, respectively. As shown in this figure, the individual averages for the entire gastric return show a greater range of variation than those representing the averages for any one particular set of samples. That is, the range of variation among the total number of titrations for the individual is greater than the variation for the collective average of the eleven sets of samples. The results of gastric analysis by this method is strikingly similar to those obtained by the "one hour method." Probably, as great variations in the acid concentration of the gastric contents would have been shown for each individual by the first method as by the third had the returned mass been divided into portions, titrated and the average ascertained. In a way this third method is simply a modification of the "one hour method," hence, it is not surprising to find that the average acid values as determined by the two methods are so closely similar. However, the point of interest for this method lies, not in the obtaining of average figures, but in the fact that by its use the acid concentration in the various portions of the stomach content can be ascertained. The degree of variation in the acid concentration of the samples returned by an individual at the end of one hour is shown in Table 2 . The average variation among all samples was free acid 16, total 25 points. The maximal variation for any one individual was free acid 35, total 65 ; the minimal variation was free acid 1 , total acid 2 points. The range of variation among individuals, therefore, was free acid 1 to 35 and for total acid 2 to 65 points. In other words, different individuals show marked variations in their power of reducing a test meal to a uniform degree of acidity in the course of one hour.

The amount of material recoverable from the stomach at the end of an hour seems to bear no constant relation to the degree of acidity of the various samples. The above figures do not indicate that the last samples withdrawn are of necessity more charged with acid than the first withdrawals, although a number of cases studied did show such a terminal increase of acid, Nos. 57 and 46. On the whole, the results of the figures as obtained by the third method show that the gastric contents at any one time may not be a homogenous mass; the acid concentration varies in different portions of the mass as a whole.

The present findings on normal men are in accord with those of Gorham who used this method of study on sixty-five patients with varied clinical diagnoses. This observer found that 73 per cent. of his cases showed a considerable variation between the first and subsequent portions withdrawn from the stomach forty-five minutes 
following a test meal. In Table 2 are shown numerically the variation in the acid values for the various portions of the returned gastric contents as determined in my series of normal young men.

Motility of the Stomach as Judged by the Amount of Gastric Return.-Roentgenographic procedures, to a large extent, have eliminated the necessity of indirect observations relative to the emptying of the stomach. However, a careful gastric analysis, to a considerable degree, is indicative of the power of the stomach to rid itself of contents.

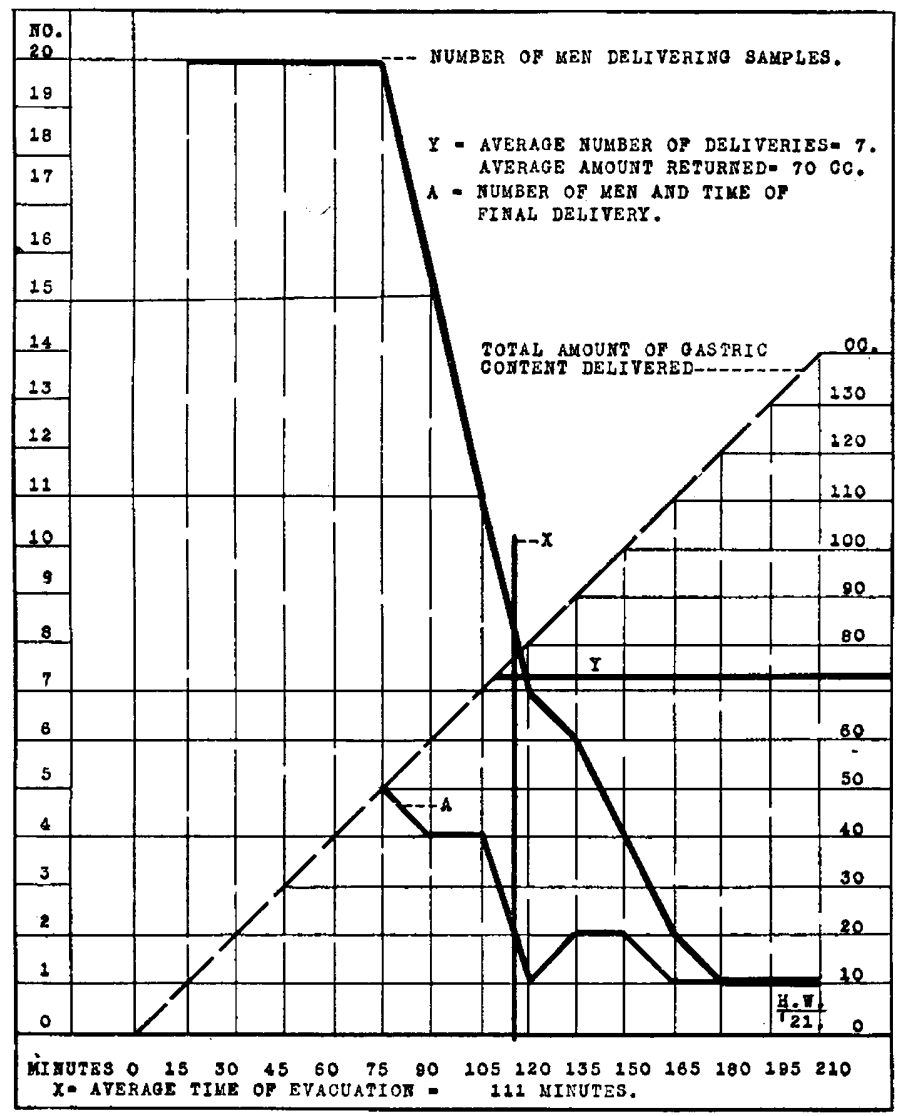

Fig. 4.-Chart showing the rate of gastric evacuation as determined by fractional withdrawals $(10$ c.c.) at 15 minute intervals. Withdrawals made every 15 minutes following ingestion of standard test meal. Observations on 20 men; 148 deliveries over a total period of 210 minutes.

The results of the present observations on this point are shown in Tables 1 and 2, and in the charts. In Group 1, twenty-eight men returned an average of 119 c. c. of gastric contents, or 23.8 per cent. of the amount ingested (Fig. 1). In other words, at the end of one hour, the twenty-eight men has passed into the duodenum 10,668 c. c., or an 
average clearance of $381 \mathrm{c.c}$., or 76.2 per cent. In Group 3, nineteen men passed during the period of one hour, $8,330 \mathrm{c}$. c. of a total of 9,500 c. c. into the duodenum; average clearance of 438 c. c. per man, or 87.6 per cent. That is, each man delivered on the average $62 \mathrm{c}$. c., i.e., 12.4 per cent., at the end of one hour. These figures show a wide variation in the amount of material that may be recovered at the end of an hour by the two methods. It is possible that in Group 1 certain men did not refrain from taking fluids prior to the test meal, also in this group the fasting content was not obtained prior to the meal. In Group 3 the fast-contents were removed prior to the meal. It is of interest to note, however, that the average free and total acids for the two groups are practically identical.

The twenty men of the second group were given a total of 10,000 c. c. of a fluid mixture and all of this, save the amount returned by aspiration, was permitted to enter the duodenum. Of this amount of fluid given $1,480 \mathrm{c}$. c. were withdrawn as samples, that is, each man averaged a return of 74 c. c., or 14.8 per cent. of the amount taken. This means that 85.2 per cent., or $426 \mathrm{c}$. c., of the material passed into the duodenum in an average period of 111 minutes. These figures when compared with those from Group 3 seem to indicate that successive withdrawals over a period of 111 minutes, 74 c.c., is the equivalent of the amount of material in the stomach at the end of one hour. In other words, successive withdrawals appear to lengthen the time for a given amount of material to pass the pylorus. This point is further emphasized by figures of Group 2, calculated for a sixty minute period. The twenty men of this group returned 800 c. c. in samples, that is, each man averaged $40 \mathrm{c}$. c. in returns, or 8 per cent. This means that 9,200 c. c., or 92 per cent., of the original 10,000 c. c. given was either in the stomach or passed into the duodenum at the end of one hour. If these figures can be compared with those in Groups 1 and 3, then it appears that successive withdrawals during the hour material is within the stomach, in some way, reduces the power of the stomach to rid itself of contents. That as much material does not leave the stomach when the "fractional method" is employed is further indicated by the fact that only in an average of 111 minutes, practically two hours, is the stomach entirfiy empty and at which time 14.8 per cent. of the material given has been withdrawn as samples. This percentage of withdrawals for 111 minutes is practically the same as the amount of material withdrawn at the end of sixty minutes by the third method. On the other hand, the average returns for both Groups 2 and 3 are less than for Group 1. It is possible that the anticipation of the tube by the first group in some way interfered with gastric evacuation. In Groups 2 and 3 this possible psychic factor was removed in that the tube was taken before the meal. 
The data presented above, relative to gastric clearance offers no direct evidence as to the mixing power of the stomach. However, the results of the "rapid withdrawal method" do, in a rough way, indicate the degree of acid concentration in various portions of the stomach contents. It further appears from the present work, and that of Gorham ${ }^{1}$ that gastric peristalsis does not act in such a manner as to cause any extensive mixing of the gastric contents as a whole. On this point the physiologist appears to hold a firmer view than the clinician who, by performing a "fractional" gastric analysis, presumes that the stomach contents is of a uniform acidity for the various phases at which samples are withdrawn for titration. Such gastric tests, though highly instructive if correctly interpreted, must be interpreted in the light of what really occurs when a sample of material is withdrawn from the stomach.

\section{REVIEW OF LITERATURE}

The modern views relative to the major activities of the stomach are well set forth by Rehfuss. ${ }^{2}$ To quote, page 28 :

During the digestive phase, the two parts, the fundus and the pyloric antrum, can be readily delineated. The fundus serves as a reservoir, receiving the food and pouring out the active gastric secretion but playing little part in active motor function. This accounts for the continuance of salivary digestion in the interior of the food mass for some time after food has left the mouth, and it explains the difference in the character of the material found in the fundus and duodenum on double gastro-duodenal intubation.

This author, after briefly reviewing the theory of "the acid control of the pylorus" and in the main accepting it, states that material is regurgitated into the stomach from the duodenum whenever the acidity of the chyme in the former reaches a high degree of concentration. To quote him on this point, pages 32-33:

(1) Trypsin is found almost constantly in both the fasting and the digestive phases of digestion, the interdigestive and digestive phases of the stomach. (2) Trypsin is highly resistant to the action of acid and pepsin and forms the index for pancreatic regurgitation. (3) The tryptic value was found to be high in the presence of low acidities and low when the contents were of high concentration. When normally the acidity reaches excessive limits, its fall is accomplished by a rise in the trypsin values. (4) We were able to demonstrate for instance, that 0.5 per cent. $\mathrm{HCl}$ is followed by a fall in acidity to about 0.2 per cent. due to the regurgitation of alkaline intestinal contents as indicated by a fall in acidity coincident with a rise in trypsin values.

For the purpose of this paper, the quotations stated above indicate that the acidity of the stomach content varies in different portions, being

1. Gorham, F. D.: Variations of Acid Concentration in Different Portions of the Gastric Chyme, and Its Relation to Clinical Methods of Gastric Analysis, Arch. Int. Med. 27:434 (Apri1) 1921.

2. Rehfuss. M. E.: Diseases of the Stomach. Oxford Medicine, Oxford Lnir. Press. Chapt. 2, pp. 19-121. 
alkaline in the fundus and inner mass of the food, and that an accumulation of acid in the pyloric portion is neutralized more or less completely at various times by the regurgitation of duodenal contents. Just what influence these factors have on the interpretation of functional secretory curves, "fractional method," is not stated by Rehfuss although considerable attention is given to a consideration of the secretory powers of the stomach in relation to diseased conditions.

In 1814. Home ${ }^{3}$ described the dog's stomach as consisting of two portions: the first, or fundic portion, containing fluid and solid material; the second, or pyloric portion, containing half digested food of uniform consistence. Eberle, in $1834,{ }^{4}$ made similar observations and further demonstrated that, when the stomach is carefully opened during digestion, the surface of the mass in the cardiac end shows signs of digestion, whereas the interior of the mass remains unchanged.

Ellenberger and Hofmeister, ${ }^{5}$ Ellenberger and Goldschmidt ${ }^{6}$ and later Scheunert ${ }^{2}$ showed that the digestive processes in the two ends of the stomach of the horse and pig are different for several hours after eating; also that different foods fed successively are not found uniformly mixed but in strata. Cannon, ${ }^{8} 1898$, by mixing bismuth subnitrate with different portions of a meal found that the various portions of the meal when eaten were arranged in layers in the stomach. The first portion of the meal arranged itself along the greater curvature, the third portion along the lesser curvature and the second portion between the other two. Ten minutes after peristalsis began, the stratification had entirely disappeared toward the pyloric end while, after a period of eighty minutes, the layers were still visible in the fundic portion.

Grützner, ${ }^{9} 1902$, after feeding a divided meal of various foodstuffs, killed the animals (frogs, cats, dogs) at various periods of time following the meal, froze the stomachs and found that only the outer layers of food were acidified and digested. The central layers in the cardiac end retained for hours a neutral or weakly alkaline reaction. Results of a similar value were obtained by Cannon ${ }^{10}$ after feeding alkalinized meals to cats and dogs.

3. Home: Lectures on Comparative Anatomy, London, 1814, 1:, p. 140.

4. Eberle: Physiologie der Verdatung, Würzburg, 1834, pp. 81, 91, 100, 154.

5. Ellenberger and Hofmeister: Arch. f. wissensch. u. prakt. Thier. 8:1882; 9:1883; 10:1884; 12:1886.

6. Ellenberger and Goldschmidt: Ztschr. f. physiol. Chem. 10:384, 1886.

7. Scheunert: Arch. f. d. ges. Physiol. 114:64, 1906.

8. Cannon, W. F.: The Mechanical Factors of Digestion, New York, 1911, Longmans, Green \& Co., p. 227; Am. J. Physiol. 1:378, 1898.

9. Grützner: Arch. f. d. ges. Physiol. 106:463, 1905 ; Deutsch. med.-Ztg. No. 28, 1902.

10. Cannon, W. B.: Am. J. Physiol. 1:379, 1898. 
Knox, ${ }^{11}$ in discussing the processes of gastric filling for man as observed radiographically, refers to the work of $\mathrm{Dr}$. Jefferson as follows :

When a small quantity of opaque food in the form of an emulsion is swallowed, it may be seen at the lower end of the esophagus, where it is momentarily held up. It then flows obliquely towards the left, where it accumulates to form a triangular-shaped shadow, with the apex pointing downward. This triangular mass continues to increase as the patient swallows more food, the time elapsing before the food passes lower down seeming to depend upon the tonicity of the stomach walls. In the next stage a narrow streak of opaque food passes down into the sinus, forming a longitudinal band filling the canalis gastricus, which lies along the lesser curvature. This stage may last only a few seconds. The sinus is gradually distended widening out from below upwards, until the whole organ is filled, except the air space at the fornix.

Modern physiology, as exemplified by textbooks, teaches that the fundic portion of the stomach exercises a steady pressure on its contents in consequence of which successive portions of the undigested food mass are presented to the walls of the stomach to replace the layers which loosened by the digestive action of the gastric juice are swept on to the pylorus by the peristaltic waves. ${ }^{8}$ An older view of the movements of the gastric contents was that described by Beaumont ${ }^{12}$ who considered that the contents of the stomach were kept in a rotary movement so as to become thoroughly mixed or churned. Possibly, the erroneous phrase "churning action of the stomach" is to be ascribed to the description by Beaumont.

Sick, ${ }^{13}$ found that materials withdrawn from the two portions of the stomach possessed different chemical and physical properties. $\mathrm{He}$ further found that coloring matter, charcoal or carmine, when taken after a semifluid meal did not appear in the pyloric region until from twenty-five to forty minutes later.

The above citations are sufficient to establish the fact that the gastric contents are not, as a rule, uniform mixtures throughout the various stages of digestion. However, I wish to call attention to several early observations which, though a century old, are in the main identical with our present theory of gastric activity.

A. P. W. Philip, ${ }^{14}$ refers to his observations on the activity of the stomach as observed in about 130 rabbits immediately after they had been killed.

11. Knox, R.: Radiography and Radiotherapeutics. New York, 1919. The Macmillan Co, pp. 328-329.

12. Beaumont, W.: Physiology of Digestion, Plattsburgh, 1833, p. 110.

13. Sick: Deutsch. Arch. f. klin. Med. 98:199, 1906.

14. Philip, A. P. W.: A Treatise on Indigestion and Its Consequences, Etc. Philadelphia, 1822, Benjamin \& Thomas Kite, p. 205. 
The first thing that strikes the eye on examining the stomach of rabbits which have lately eaten is, that the new is never mixed with the old food. The former is always found in the center, surrounded on all sides by the old food, except that on the upper part between the new food and the smaller curvature of the stomach there is sometimes little or no old food. All around, the nearer the food lies to the surface of the stomach the more it is digested. This is true even with regard to the food in the small curvature, compared with that nearer the center, and the food which touches the surface of the stomach is more digested than any other found in the same part of the stomach; but, unless the animal has not eaten for a great length of time, the food in contact with the surface of the stomach is in very different stages of digestion in different parts of the organ. It is least digested in the small curvature, more in the large end, and still more in the middle of the great curvature. The foregoing observations apply to the cardiac portion of the stomach; the food in the pyloric portion is always found in a state very different from that just described. It is more equally digested, the central parts differing less from those which lie near the surface of the stomach. It is evident however, that all the change effected in the stomach is not complete when the food enters this portion of it, because we find it the more digested the nearer it approaches to the pylorus, where, being ready to pass into the intestine, it has undergone all that part of digestion which is performed in the stomach. It appears, that in proportion as the food is digested it is moved along the great curvature, where the change in it is rendered more perfect to the pyloric portion. Thus the layer of food lying next the surface of the stomach is first digested, and in proportion as this undergoes the proper change, and is moved on by the muscular action of the stomach, that next in turn succeeds to undergo the same change. As the gastric fluid, to a certain extent, pervades the contents of the stomach, though apparently in no other way than by juxtaposition, for the arrangement of the food, above described we never found disturbed; the change in each part, which in its turn comes in contact with the stomach, is far advanced before it is in actual contact with it; and consequently is soon after this in a proper state to be moved on towards the pyloric end. Thus a continual motion is going on, that part of the food which lies next the surface of the stomach passing towards the pylorus, and the more central parts approaching the surface.

The observations and explanations offered by Philip concerning the activities of the stomach were both warmly received and rejected by his contemporaries. For instance, J. A. Paris, ${ }^{16}$ O. Halstead, ${ }^{15}$ and James Johnson ${ }^{17}$ quote the works of Philip freely and agree with his findings. On the other hand, Nathan R. Smith, ${ }^{18}$ writing three years after the publication of Philip's work, although quoting this author, attacks the entire theory of secretory functioning of the stomach as evolved by Spallanzani, Hunter and others. The vehemence of this attack by Smith is shown in the following quotation, page 25.

15. Halstead, O.: A Full and Accurate Account of the New Method of Curing Dyspepsia, Discovered and Practiced by O. Halstead. New York, 1830 , p. 156.

16. Paris, J. A.: A Treatise on Diet, Etc. New York, 1828, E. Duyckinck, Collins \& Co., Collins \& Hannay, and O. A. Roorbach, p. 210.

17. Johnson, James: An Essay on Indigestion, Etc. Philadelphia, 1831, Nathan Kite, p. 194.

18. Smith, Nathan R.: A Physiological Essay on Digestion, New York, 1825, E. Bliss and E. White, p. 93. 
It were ridiculous in us, as it has appeared in many writers on this subject, to inflict, like Falstaff, a new stab on these dead theories for the sake of making the victory, in part, our own. They are obviously inconsistent with the principles of medical logic, now well established.

\section{Further.}

The sensible properties of chyme suggested to some of the older anatomists the idea that the aliment was, in the stomach, attacked by a myriad of small worms which reduced it to the uniform pulpy mass of this substance. Perhaps they were as near the truth as those who consider the process to be performed by a chemical agent, for neither the existence of these wonderful worms, nor of the less wonderful fluid can be proved, and if we reason from the effect alone, we should with more propriety ascribe it to the former.

Reaumur, Spallanzani, Hunter, Magendie, Fordyce, Prout, Philip and Paris to the contrary, one might add. However, the observations of Philip have in principle withstood adverse criticism.

\section{DISCUSSION}

From the foregoing references to the literature and from the results of a limited number of gastric analyses it appears that the gastric content is not of a uniform acid concentration and that the stomach does not act as a churn or general mixing apparatus. If such conditions are to be accepted as normal then it appears that any single sample of the gastric content at any time following the taking of a meal, of necessity, will not be representative of the remaining content. A given sample of the gastric contents can only be of value when it is definitely known from which portion of the stomach it was removed.

A correct analysis of gastric activity can only be made when it is known (1) from what portion of the stomach the tube is delivering; (2) whether or not duodenal regurgitation is occurring; (3) the degree of motility of the stomach; (4) the secretory power of the stomach and, (5) the effects of withdrawals on the motility and secretory functions. If the works of Rehfuss, Hawk, Boldyreff and others are correct, and we have no reason to discredit them at the present time, then the position of the stomach tube tip becomes of relatively little importance for the reason that a tip in the pyloric antrum may deliver not only samples of high acid concentration but also samples of low acid concentration and high tryptic value because of duodenal regurgitation. Gastric motility may also shift the tube tip about in such a manner as to cause the delivery of material from any portion of the stomach. The rapidity with which the stomach empties itself might also alter the degree of acid concentration for any set of samples withdrawn. It further appears that the response of a given stomach to a test meal should also be considered, as recent work has shown that the same stomach responds differently to different types of foodstuffs. The usual test meal, especially the one used in the present experimental work, is far 
from palatable even though it allay hunger contractions. Lastly, as pointed out above, the effects of the sudden withdrawal of 10 c.c. amounts from the stomach at stated intervals should be considered. It is not justifiable, at least at the present time, to assume that the removal of such quantities from the stomach is in any way the equivalent to the ejection of chyme through the pyloric sphincter into the duodenum. The tonicity of the stomach, especially of the fundic end, is usually just sufficient to approximate the walls of the stomach against the mass contained. Under such a degree of tonicity the fundus presents new material to the pyloric region, small portions of which are expelled more or less rhythmically into the duodenum. Hence, it appears that a sudden reduction in the mass within the stomach would result in at least a temporary relative loss of tonicity, i.e., the stomach would have to reinstate a tonicity sufficient to exert a pressure on the gastric mass. This point seems to be of some interest although the figures given above are not sufficient to establish the explanation given.

From the above considerations it becomes evident that "correlation of all data is essential to successful interpretation of gastric curves." : Further than this, it appears that gastric curves, in a majority of cases, cannot justly be taken to represent the powers of the stomach to form acid from any point in the curve cannot be taken as indicative of the mass as a whole left in the stomach at that time of withdrawal. In the words of Cannon, ${ }^{8}$ who quotes Sick, Prym et al.: "Evidently, if the contents are not a uniform and homogenous mixture, not only may the stomach tube give wrong testimony, but the food even when expressed as a whole be equally deceptive." When added to this we find duodenal regurgitation with its consequent reduction of the acid concentration in the pyloric portion of the stomach, the chances for "wrong testimony" become greatly increased.

In spite of the fact that the present work tends to diminish the importance of the "fractional method," it is not the desire to leave the impression that gastric analyses are of but little clinical importance. It is true that "The use of the roentgen ray and exploratory incision," as suggested by Green, " "has displaced the systematic examination of the gastric contents to an unjustifiable degree. In relation to the stomach the tube is the most valuable of our aids to diagnosis and therapy alike."

SUMMART AND CCNCLLSFON

1. The acid concentration of the gastric contents is not, in the majority of cases (nineteen young men), constant in all portions of the gastric content at the end of one hour of digestion.

19. Green, C. L.: Medical Diagnosis for the Student and Practitioner, Philadelphia, P. Blakiston's Son \& Co. 
2. The withdrawal of gastric contents for the purpose of determining the acid concentration, the type of the meal, the position of the tube tip, and duodenal regurgitation are factors which militate against the acceptance of "Fractional Curves" as indicative of the secretory functions of the stomach. The variations in the curves of the twenty men studied by the "fractional method" may, in part, be the result of these physiologic factors.

3. As pointed out by Gorham these physiologic factors, in great part may be held as responsible for the various "Secretory Curves" which formerly were considered functional alterations in the power of the stomach to form acid. 